\title{
Aberrant DNA methylation and expression of ERAP1 gene in ankylosing spondylitis
}

\author{
Yubo Ma ${ }^{1}$, Dazhi Fan ${ }^{2}$, Shanshan $\mathrm{Xu}^{1}$, Jixiang Deng ${ }^{1}$, Xing Gao ${ }^{1}$, Xu Zhang ${ }^{1}$, and faming \\ $\operatorname{pan}^{3}$ \\ ${ }^{1}$ Anhui Medical University \\ ${ }^{2}$ Southern Medical University Affiliated Maternal and Child Health Hospital of Foshan \\ 3 anhui medical university
}

May 15, 2020

\begin{abstract}
Summary Objective: Endoplasmic reticulum aminopeptidase 1 (ERAP1) is known to participate in the pathogenesis of ankylosing spondylitis (AS) cooperated with HLA-B27. This study aimed to evaluate the relationship between promoter methylation and mRNA levels of ERAP1 and AS. Methods: The DNA methylation level of 100 AS patients and 100 health controls (HCs) were tested using targeted bisulfite sequencing assay. Besides, the mRNA level of 20 AS patients and HCs was measured used quantitative real-time reverse transcription-polymerase chain reaction to verify the results of DNA methylation. Results: The methylation levels of two CpG islands containing 31 loci in ERAP1 promoter were measured. ERAP1_1 (P < 0.001) and ERAP1_2 $(\mathrm{P}<0.001)$ islands were significantly hyperrmethylated in AS patients compared with healthy controls. Correspondingly, the mRNA level was significantly lower in AS patients. The ROC curve analysis reported the sensitivity, specificity and area under curve were $0.717,0.737$ and 0.779 of differential methylated CpG loci of ERAP1 for AS diagnosis. Besides, we also found that the methylation level was associated with the family history, non-steroidal anti-inflammatory drugs use, X-ray classification and clinical manifestations. Conclusions: Our study demonstrated that the ERAP1 gene is significantly hypermethylated in AS patients, which is verified by the lower mRNA level of AS patients. Our findings suggested that aberrant methylation of ERAP1 promoter may take part in the pathogenesis of AS and can be considered as diagnostic tool and therapeutic target of AS.
\end{abstract}

\section{Aberrant DNA methylation and expression of ERAP1 gene in ankylosing spondylitis}

Running title: ERAP1 methylation in AS

Authors: Yubo Ma ${ }^{\mathrm{a}, \mathrm{b}}, \mathrm{MM}$, Dazhi Fan ${ }^{\mathrm{c}}$, MM, Shanshan $\mathrm{Xu}^{\mathrm{a}, \mathrm{b}}, \mathrm{MM}$, Jixiang Deng ${ }^{\mathrm{a}, \mathrm{b}}$, MM, Xing Gao ${ }^{\mathrm{a}, \mathrm{b}}$, MM, Xu Zhang a,b, MD, Faming Pan ${ }^{a, b, \#, ~ M D ~}$

Affiliations: a Department of Epidemiology and Biostatistics, School of Public Health, Anhui Medical University, Hefei, Anhui, China; b The Key Laboratory of Major Autoimmune Diseases, Anhui Medical University, 81 Meishan Road, Hefei, Anhui, China; c Foshan Institute of Fetal Medicine, Southern Medical University Affiliated Maternal and Child Health Hospital of Foshan, Foshan, Guangdong, China.

Corresponding Author: Faming Pan, Department of Epidemiology and Biostatistics, School of Public Health, Anhui Medical University, Hefei, Anhui, China. E-mail:famingpan@ahmu.edu.cn

Key words: ankylosing spondylitis; DNA methylation; ERAP1; mRNA

Abbreviations: AS: ankylosing spondylitis; AUC: area under curve; BASDAI: Bath Ankylosing Spondylitis Disease Activity Index; BASFI: Bath Ankylosing Spondylitis Functional Index; CI: confidence interval; CpG: 
cytosine-guanine dinucleotide; CRP: C-reactive protein; ERAP1: endoplasmic reticulum aminopeptidase 1; ESR: erythrocyte sedimentation rate; EWAS: epigenome-wide association study; HLA: human leukocyte antigen; OR: odds ratio; PCR: polymerase chain reaction; ROC: Receiver operating characteristic; NSAIDs: Non-steroidal anti-inflammatory drugs.

\section{Summary}

Objective : Endoplasmic reticulum aminopeptidase 1 (ERAP1) is known to participate in the pathogenesis of ankylosing spondylitis (AS) cooperated with HLA-B27. This study aimed to evaluate the relationship between promoter methylation and mRNA levels of ERAP1 and AS.

Methods : The DNA methylation level of 100 AS patients and 100 health controls (HCs) were tested using targeted bisulfite sequencing assay. Besides, the mRNA level of $20 \mathrm{AS}$ patients and HCs was measured used quantitative real-time reverse transcription-polymerase chain reaction to verify the results of DNA methylation.

Results : The methylation levels of two CpG islands containing 31 loci in ERAP1 promoter were measured. ERAP1_1 $(P<0.001)$ and ERAP1_2 $(P<0.001)$ islands were significantly hyperrmethylated in AS patients compared with healthy controls. Correspondingly, the mRNA level was significantly lower in AS patients. The ROC curve analysis reported the sensitivity, specificity and area under curve were $0.717,0.737$ and 0.779 of differential methylated CpG loci of ERAP1 for AS diagnosis. Besides, we also found that the methylation level was associated with the family history, non-steroidal anti-inflammatory drugs use, X-ray classification and clinical manifestations.

Conclusions : Our study demonstrated that the ERAP1 gene is significantly hypermethylated in AS patients, which is verified by the lower mRNA level of AS patients. Our findings suggested that aberrant methylation of ERAP1 promoter may take part in the pathogenesis of AS and can be considered as diagnostic tool and therapeutic target of AS.

\section{Highlights}

1) In the article we have evaluated the promoter methylation levels of two $\mathrm{CpG}$ islands containing 31 loci in ERAP1 gene. The methylation levels of ERAP1_1 $(\mathrm{P}<0.001)$ and ERAP1_2 $(\mathrm{P}<0.001)$ islands were significantly hypermethylated in AS patients compared with healthy controls.

2) Correspondingly, the mRNA level of EARAP1 was significantly lower in AS patients.

3) The ROC curve analysis also reported that the sensitivity, specificity and area under curve were 0.717 , 0.737 and 0.779 of 15 differential methylated CpG loci of ERAP1 for AS diagnosis.

4) Besides, we also found that the methylation level was associated with the family history, non-steroidal anti-inflammatory drugs use, X-ray classification and clinical manifestations of AS.

\section{Introduction}

Ankylosing spondylitis (AS) is a common chronic rheumatic arthritis, characterized with progressive bone proliferation of axial skeleton and sacroiliac joints. The exactly pathogenesis of AS is still obscure now, but studies indicated that gene and environmental interaction plays roles in the development and progression of AS (1,2). Twins and family based studies estimated the heritability of AS is about $90 \%(2-4)$. The human leukocyte antigen (HLA)-B27, which is encoded in class 1 major histocompatibility complex region, is the strongest risk factor of AS (4). Recent genome-wide association and case-control studies indicated that endoplasmic reticulum aminopeptidase 1 (ERAP1) is significant associated with AS through cooperating with HLA-B27 (5). The total of 114 loci are established to associate with AS; however these genetic variants are reported to only accounting for about $30 \%$ of genetic risk (6). Recent studies indicated that epigenetics may partly account for the inter-individual variance of heterogeneity.

DNA methylation as a most common reported epigenetic modification plays pivotal roles in various life courses, as growth and differentiation, through programmed gene expression regulation in the genome. DNA 
methylation is the addition of a methyl group to 5' position of a cytosine DNA base in the middle of cytosine-guanine dinucleotide (CpG) (7). The abnormal DNA methylation in the gene promoter is generally associated with transcriptional silencing and linked to ranges of diseases $(8,9)$. Recently, increasing number of epigenome-wide association studies (EWAS) indicated that DNA methylation plays pivotal roles in the mechanism of rheumatic diseases as systematic lupus erythematosus, rheumatoid arthritis, and AS (10-14). One EWAS found 1915 altered DNA methylation loci of AS. Besides, candidate targeted gene methylation studies also reported differential methylation loci of AS patients. Methylation of SOCS-1 gene was detected in serum of HLA-B27 positive AS patients but not B27 positive controls, and significantly associated with higher serum cytokines and severity of clinical manifestations of AS patients (15). Hypermethylation and decreased expression of DNMT1 and BCL11 B genes were both reported to associate with AS $(16,17)$. Nevertheless, study focus on DNA methylation and AS is still scarce and urgent.

ERAP1 is a polymorphic aminopeptidase within the endoplasmic reticulum, known as "molecular ruler" to trim peptides to nine amino acids in length for binding to HLA class I molecules on antigen-presenting cells for subsequent interaction with $\mathrm{CD} 8^{+} \mathrm{T}$ cells $(18,19)$. Recent single nucleotide polymorphism studies of our team and other scholars also proved that ERAP1 plays pivotal roles in the pathogenesis of AS through cooperating with HLA-B27 $(5,14)$. Taken into account of these factors, we designed a two stage casecontrolled study to evaluate the promoter methylation and transcriptional profile of $E R A P 1$ gene, respectively, in peripheral blood mononuclear cells of AS patients and healthy controls (HCs).

\section{Materials and methods}

\section{Study populations}

A total of 100 AS patients and 100 age and gender matched HCs were enrolled in the DNA methylation examination stage, and 20 patients and 20 controls were recruited in the mRNA expression verification stage. All patients and HCs were recruited from the Department of Rheumatology at the First Affliation Hospital of Anhui Medical University. Diagnosis of AS was made by qualified rheumatologists according to the modified New York criteria (20). Blood donors with no history of rheumatic diseases or other chronic diseases were included as controls. DNA and mRNA samples were extracted from the $5 \mathrm{ml}$ peripheral venous blood of all participants. Besides, all patients have filled out a questionnaire about the general geographic and clinical characteristics. Detailed clinical indicators as medication use, HLA-B27, C-reactive protein (CRP), erythrocyte sedimentation rate (ESR), Bath Ankylosing Spondylitis Disease Activity Index (BASDAI) and Bath Ankylosing Spondylitis Functional Index (BASFI) were reported. This study was approved by the ethics committee of Anhui Medical University with the serial number of 20170225. All patients and controls provided their written informed content.

\section{Targeted bisulfite sequencing assay}

CpG islands in the proximal promoter of ERAP1 gene were examined from $2 \mathrm{k}$ bp upstream of transcript start site to $1 \mathrm{k} \mathrm{bp}$ downstream of the first exon satisfying the following criteria: a) observed to expected ratio of $\mathrm{CpG}$ dinucleotide $>0.60 ; \mathrm{b}$ ) percentage of cytosine and guanine $>50 \%$; c) length $>200 \mathrm{bp}$. DNA sequences of the CpG islands in ERAP1 promoter region were determined by an online database of the University of California, Santa Cruz (http://www.genome.ucsc.edu), and the primers sequences for ERAP1 methylation were designed by the EpiDesigner online software (http:// www.epidesigner.com) accordingly. And 31 methylation sites of two CpG islands (ERAP1_1 and ERAP1_2) were analyzed in our study. The primers sequence of ERAP1_1 and ERAP1_2 methylation were listed as following: ERAP1_1, forward: 5'AGGGTTAGGGGTATGTAGGAAAG-3', reverse: 5'- CCTTCCTCCTCTACAACATCTCC-3'; ERAP1_2, forward: 5'- GTTTTGGGGTYGTTTTTATTTTTG-3', reverse: 5'- TTACCCTTTCCCCAACTCC -3'.

Genomic DNA was firstly extracted from peripheral venous blood of participants using DNeasy Blood Tissue Kit QIAGEN Kit (QIAGEN, Germany) in line with manufacturer's protocol, and quantified and qualified through NanoDrop ${ }^{\mathrm{TM}} 2000$ Spectrophotometer (Thermo Fisher Scientific, Wilmington, DE, USA.). Genomic DNA (400 ng) was bisulfite converted using EZ DNA Methylatio ${ }^{\text {TM }}$-GOLD Kit (Zymo Research, Irvine, USA). Multiplex polymerase chain reaction (PCR) was performed with above primers combination, and 
PCR amplicons were separated and purified through agarose electrophoresis and QIAquick Gel Extraction kit (QIAGEN, Germany). Corresponding libraries were sequenced on Illumina NextSeq platform according to manufacturer's protocols.

\section{Quantitative real-time PCR assay}

Peripheral blood mononuclear cells were isolated from peripheral blood using density gradient centrifugation. Then, total RNA were extracted and purified from peripheral blood mononuclear cells by TRIzol ${ }^{\text {TM }}$ LS reagent. Total RNA was reverse translated as complementary DNA after the degradation of mixed genomic DNA using PrimeScript ${ }^{\mathrm{TM}}$ RT reagent Kit with gDNA Eraser (Takara Bio Inc., Japan). SYBR Green kit (Takara Bio Inc., Japan) was used in quantitative real-time PCR process based on QuantStudio ${ }^{\mathrm{TM}} 7$ Flex (Life Technologies, USA). The expression data of ERAP1 was normalized to internal reference $\beta$-actin, and the relative expression level of ERAP1 was calculated by comparative $2^{-\Delta \Delta}{ }^{\prime \prime} \tau$ method. Primers sequence of ERAP1 and $\beta$-actin were listed as following: $\beta$-actin, forward: 5'-CTCCATCCTGGCCTCGCTGT-, reverse: 5'-GCTGTCACCTTCACCGTTCC-; ERAP1, forward: 5'-TTTGAACTTGGCTCATCTTCC-, reverse: 5'AATTGTCTGTTGGACACAACG .

\section{Statistical analysis}

The distribution of the included variables of our study was tested via Shapiro- Wilk method. Normal distribution data was presented as mean \pm standard variance, otherwise median and (interquartile range) was presented. Correspondingly, student's t test or Mann-Whitney U test were used to test the between group difference of the mean or mean rank, respectively. Spearman's rank correlation efficiency was test to evaluate the association between ERAP1 methylation level and other continuous or hierarchical variables. Univariate logistic regression analysis was used to calculate the odds ratio (OR). Odds ratio (OR) with $95 \%$ confidence interval (CI) and forest plot were used to evaluated association of individual methylation site and AS visualized. Multiple logistic regression analysis was used to establish a regression model. Receiver operating characteristic (ROC) curve and area under curve (AUC) were applied to assess the predictive value of the ERAP1 methylation as a biomarker of AS. All these data analysis diagram plotting were accomplished by SPSS 23.0 software (SPSS Inc., Chicago, IL, USA), GraphPad Prism 7.00 (GraphPad Software Inc., CA, USA) and R software. A two-tailed $P<0.05$ was taken as statistically significant, and multiple comparisons were adjusted through Bonferroni method.

\section{Results}

\section{Characteristics of participants}

In the candidate targeted gene methylation detection stage, of the enrolled 100 patients and $100 \mathrm{HCs}$, one patient and one $\mathrm{HC}$ failed in bisulfite conversion assay. Detailed demographic and clinical characteristics were listed in Table 1. There were no significant difference in gender $\left(\chi^{2}=1.172, P=0.279\right)$ and mean age $(\mathrm{t}=0.564, P=0.574)$ existed between patients and HCs. In the second stage, 20 patients and 20 HCs were tested the mRNA level of ERAP1 gene. Similarly, no any significant difference in mean age $(\mathrm{t}=$ $0.102, P=0.919)$ and gender $\left(\chi^{2}=0.125, P=0.723\right)$ were found. Data medication use and unilateral X-ray classification were only retrieved from a proportion of AS patients.

\section{DNA methylation of ERAP1 gene}

In total, two $\mathrm{CpG}$ islands containing 31 loci were tested the proportion of methylated cytokine accounting for the total cytokine. Among the 15 loci of ERAP1_1 island and 16 loci of ERAP2 island, 13 CpG sites, ERAP1_1_46, ERAP1_1_91, ERAP1_1_107, ERAP1_1_118, ERAP1_1_222, ERAP1_2_28, ERAP1_2_31, ERAP1_2_46, ERAP1_2_49, ERAP1_2_69, ERAP1_2_95, ERAP1_2_121 and ERAP1_2_136, were significant hypermethylated in AS patients compared to HCs used Mann-Whitney U test detailed in Table 2. Logistic regression also indicated that methylation of six loci in ERAP1_2 island, ERAP1_2_28, ERAP1_2_31, ERAP1_2_49, ERAP1_2_69, ERAP1_2_121 and ERAP1_2_136, were significantly associated with increased risk of AS visualized in Figure 1A. Among them, ERAP1_2_28 located at chr5: 96143595 was reported to 
have highest effect size with OR $=5.390$ (95\% CI: 2.048 to 14.184$)$ of as a percent of total cytokine methylated. The average methylation levels of ERAP1_1 $(Z=-4.831, P<0.001)$ and ERAP1_2 $(Z=-6.140, P$ $<0.001$ ) islands were also calculated to associated with AS (Figure 1B \& 1C). ROC curve analysis on the average methylation levels of the two CpG islands also indicated their potential as biomarkers of AS. The AUC of ROC curve for ERAP1_1 island was 0.669 (95\% CI: 0.626 to 0.707, $P<0.001$ ), with a sensitivity of 0.818 and a specificity of 0.531 for the best cutoff point 0.671 (Figure 1D, Table 3). Correspondingly, the AUC of ERAP1_2 was 0.750 (95\% CI: 0.683 to $0.818, P<0.001$ ), and the best cutoff point was 0.681 , with a sensitivity of 0.707 and specificity of 0.684 (Figure 1D, Table 3). We also established a regression model included the above mentioned 13 significant CpG loci detailed in Table S1, with each sample obtained a probability value. ROC curve analysis on the probability value also reported that the AUC was $0.779(95 \%$ CI: 0.714 to $0.844, P<0.001)$, and best cutoff point was 0.500 , with a sensitivity of 0.717 and specificity of 0.737 (Figure 1D, Table 3).

\section{Subgroup and correlation analyses and of ERAP1 methylation}

ERAP1 was known to play roles cooperated with HLA-B27 in AS. Nevertheless, we found that the methylation levels of ERAP1_1 and ERAP1_2 islands of HLA-B27 positive patients were not significantly different from HLA-B27 negative patients $(Z=-0.180, P=0.857)$. The known environmental factors associated with DNA methylation of smoking $(Z=-0.268, P=0.789)$ and alcohol use $(Z=-0.969, P=0.332)$ were also reported not associated with the methylation level in AS patients detailed in Table 4. Among the demographic and clinical factors, we found that patients with family history have lower methylation level of ERAP1_1 island $(Z=-2.258, P=0.024)$. Non-steroidal anti-inflammatory drugs (NSAIDs) use was significantly associated with higher methylation level of ERA1_2 island $(Z=-2.113, P=0.035)$, but not any medication use history $(Z=-0.605, P=0.545)$. As to the variates about disease activity and function, we also found that the methylation level of ERAP1_1 island was significant associated with global back pain $\left(\mathrm{r}_{\mathrm{s}}=0.502, P=0.002\right)$ of AS patiens. X-ray classification of sacroiliac joint $\left(\mathrm{r}_{\mathrm{s}}=0.548, P=0.018\right)$ was associated with the methylation level of ERAP1_2 island (Table 5).

\section{Expression level and correlation analysis of ERAP1 mRNA}

In order to verify the differential methylation level of ERAP1, we also measured the mRNA expression level in $20 \mathrm{AS}$ patients and $20 \mathrm{HCs}$. The expression level of ERAP1 was significant decreased in AS patients compared with HCs $((Z=-4.048, P<0.001$, Figure $1 \mathrm{e})$. In the correlation analysis, we also found that body mass index $\left(\mathrm{r}_{\mathrm{s}}=-0.659, P=0.002\right)$, chest expansion $\left(\mathrm{r}_{\mathrm{s}}=0.697, P=0.001\right)$ and Schober test score $\left(\mathrm{r}_{\mathrm{s}}=0.537, P=0.018\right)$ were associated with ERAP1 level.

\section{Discussion}

Our study first proved that the relationship betweenERAP1 and AS from the epigenetic aspect. The result proved that hypermethylation ofERAP1 promoter in the peripheral blood was associated with AS. The function and disease activity index and NSAIDs were also significantly associated with the methylation level. Correspondingly, the mRNA expression level was also proved to significantly decrease in AS patients.

Unlike the DNA text, the sequence of nucleotides, containing the genetic information, the annotation system of chemical modification was exited for instructing how and when to read the text in mammals. DNA methylation plays sophisticated roles in annotating genetic information. The existence of DNA methylation in gene regulatory regions, as promoter and enhancer, would recruit a group of factors programmed generating a closed chromatin structure and consequently repressing the gene expression. Our study reported that the DNA methylation of ERAP1 was associated with AS, consistent with results of previous studies thatEARP1 plays pivotal roles in the pathogenesis of AS. In the verification stage, we also found that the mRNA expression level of AS patients were significantly deceased, which is consistent with the result that ERAP1 gene was hypermethylated in AS patients.

Different form the sequence of DNA, previous study has proved that DNA methylation patterns derived from the gametes will erased before embryo implantation and the new methylation profile will established in 
each mammal $(21,22)$. Intriguingly, we found something unusual that ERAP1_1 in AS patients with family history were hypomethylated. One reason may be that patients with family history inherited higher risk of AS, and the pathogenesis of AS propositus was more like to the results of exposure of environmental risk factors, which made them have higher methylation level than patients with higher inherited risk of AS. Even though ERAP1 was established to cooperate with HLA-B27 in AS, the results indicated that HLA-B27 status may not associated with the methylation level of ERAP1 in AS patients. Consistently, previous studies also indicated that family history was associated with methylation level of diseases related genes $(23,24)$. Our study also suggested that NSAIDs was associated with the methylation of ERAP1_1, and the tumor necrosis factor inhibitor and sulfasalazine were irrelevant with the methylation level. Similarly, previous study also proved that celecoxib could reverse the DNA hypomethylation status in rat colon tumors. This study also indicated that preventive efficacy of various agents may be the results of their effect on reverse DNA hypomethylation in some extent (25). Our results also indicated that the efficacy of NSAIDs on repression the progression of ossification may be the results of epigenetic regulation of corresponding genes. However, the nature of cross-sectional design our study make us only can provide association result. The result should be verified by prospective study, and further study about the underlying mechanism was also helpful.

In the correlation analysis, we also found that the methylation level of ERAP1_2 was positively associated with the X-ray classification of sacroiliac joint. Besides, we also found that the mRNA level ofERAP1 was positively associated the thoracic and lumbar mobility. It seemed that ERAP1 was more associated with the long-standing joint ossification. And the higher methylation and corresponding lower mRNA levels of ERAP1 was associated with severer ossification. These results were consistent with the conclusion that ERAP1 were associated with AS. Besides, we also found something interesting that body mass index was positively associated with the mRNA level ofERAP1. Relevant study was still devoid, and the relationship should be verified in further with larger sample size. The ROC curve analysis indicated that the ERAP1 methylation level could serve as biomarkers, by either $\mathrm{CpG}$ island or entire promoter, to distinguish AS patients from HCs.

Our study has several strengths. To best of our known, this study was the first research reported the relationship between ERAP1 methylation status and AS and verified the mRNA expression level ofERAP1 . Besides, we have also evaluated the relationship between various environmental factors, clinical manifestations or medication use and the methylation level. Considering the less invasiveness and higher diagnostic efficiency, methylation test was valuable in clinical setting for AS diagnosis. Recent study indicated that drugs as celecoxib was could prevent diseases as tumor (25), which suggested the treatment potential of agents specially regulated the methylation factors of ERAP1. The finding that NSAIDs use was positive associated the methylation level of ERAP1_1 also indicated the treatment efficacy of NSAIDs on AS was via altering the methylation level of corresponding genes in some extent.

These are also some limitation should be considered. First, subjects for the testing of DNA methylation and mRNA were two separate groups, so the relationship analysis between DNA methylation and mRNA was not applicable. Second, the methylation profiles were different across tissues and cell subtypes. ERAP1 methylation level of peripheral blood mononuclear cells can not present the methylation of specific cell. In the end, the single center case-control study design limited the generalizability of results, and further lager scale prospective study and animal model research are necessary.

\section{Conclusions}

In summary, our study demonstrated that the ERAP1 is significantly hypermethylated in AS patients, and the result is also verified by the lower mRNA level of AS patients. Our findings suggested that aberrant methylation of ERAP1 promoter may take part in the pathogenesis of AS and can be considered as diagnostic tool and therapeutic target of AS. The finding that NSAIDs use could alter the methylation level indicated that the efficacy of NSAIDs on AS could be through altering the methylation level of corresponding genes in some extent.

\section{Acknowledgement}


We thank all the patients and healthy controls who participated in our study.

\section{Authors' contributions}

Faming Pan and Yubo Ma executed the research work and are accountable for the data quality and accuracy. Faming Pan and Yubo Ma contributed to the study design, data acquisition, statistical analysis and draft manuscript. Dazhi Fan, Shanshan Xu, Jixiang Deng, Xing Gao and Xu Zhang prepared manuscript preparation, acquired data and diagnosed patients. All authors had access to the extracted data in the study and agree to the conclusions made. All authors approved the manuscript.

\section{Funding}

This study was supported by the National Natural Science Foundation of China [grant numbers 81773514, 81573218 and 81273169].

\section{Ethics approval}

The study was approved by the Local Ethics Research Committee of Anhui Medical University, and all participants provided their written informed content.

\section{Conflict of interest}

None declared.

\section{References}

1. Cortes A, Hadler J, Pointon JP, Robinson PC, Karaderi T, Leo P, Cremin K, Pryce K, Harris J, Lee S, Joo KB, Shim SC, Weisman M, Ward M, Zhou X, Garchon HJ, Chiocchia G, Nossent J, Lie BA, Forre O, Tuomilehto J, Laiho K, Jiang L, Liu Y, Wu X, Bradbury LA, Elewaut D, Burgos-Vargas R, Stebbings S, Appleton L, Farrah C, Lau J, Kenna TJ, Haroon N, Ferreira MA, Yang J, Mulero J, Fernandez-Sueiro JL, Gonzalez-Gay MA, Lopez-Larrea C, Deloukas P, Donnelly P, Bowness P, Gafney K, Gaston H, Gladman DD, Rahman P, Maksymowych WP, Xu H, Crusius JB, van der Horst-Bruinsma IE, Chou CT, Valle-Onate R, Romero-Sanchez C, Hansen IM, Pimentel-Santos FM, Inman RD, Videm V, Martin J, Breban M, Reveille JD, Evans DM, Kim TH, Wordsworth BP, Brown MA. Identification of multiple risk variants for ankylosing spondylitis through high-density genotyping of immune-related loci. Nat Genet 2013;45:730-738.

2. Pedersen OB, Svendsen AJ, Ejstrup L, Skytthe A, Harris JR, Junker P. Ankylosing spondylitis in Danish and Norwegian twins: occurrence and the relative importance of genetic vs. environmental effectors in disease causation. Scand J Rheumatol 2008;37:120-126.

3. Brown MA, Kennedy LG, MacGregor AJ, Darke C, Duncan E, Shatford JL, Taylor A, Calin A, Wordsworth P. Susceptibility to ankylosing spondylitis in twins: the role of genes, HLA, and the environment. Arthritis Rheum 1997;40:1823-1828.

4. Calin A, Marder A, Becks E, Burns T. Genetic differences between B27 positive patients with ankylosing spondylitis and B27 positive healthy controls. Arthritis Rheum 1983;26:1460-1464.

5. Evans DM, Spencer CC, Pointon JJ, Su Z, Harvey D, Kochan G, Oppermann U, Dilthey A, Pirinen M, Stone MA, Appleton L, Moutsianas L, Leslie S, Wordsworth T, Kenna TJ, Karaderi T, Thomas GP, Ward MM, Weisman MH, Farrar C, Bradbury LA, Danoy P, Inman RD, Maksymowych W, Gladman D, Rahman P, Morgan A, Marzo-Ortega H, Bowness P, Gaffney K, Gaston JS, Smith M, Bruges-Armas J, Couto AR, Sorrentino R, Paladini F, Ferreira MA, Xu H, Liu Y, Jiang L, Lopez-Larrea C, Diaz-Pena R, Lopez-Vazquez A, Zayats T, Band G, Bellenguez C, Blackburn H, Blackwell JM, Bramon E, Bumpstead SJ, Casas JP, Corvin A, Craddock N, Deloukas P, Dronov S, Duncanson A, Edkins S, Freeman C, Gillman M, Gray E, Gwilliam R, Hammond N, Hunt SE, Jankowski J, Jayakumar A, Langford C, Liddle J, Markus HS, Mathew CG, McCann OT, McCarthy MI, Palmer CN, Peltonen L, Plomin R, Potter SC, Rautanen A, Ravindrarajah R, Ricketts M, Samani N, Sawcer SJ, Strange A, Trembath RC, Viswanathan AC, Waller M, Weston P, Whittaker P, Widaa S, Wood NW, McVean G, Reveille JD, Wordsworth BP, Brown MA, Donnelly 
P. Interaction between ERAP1 and HLA-B27 in ankylosing spondylitis implicates peptide handling in the mechanism for HLA-B27 in disease susceptibility. Nat Genet 2011;43:761-767.

6. Hanson A, Brown MA. Genetics and the Causes of Ankylosing Spondylitis. Rheum Dis Clin North Am 2017;43:401-414.

7. Zhu H, Wang G, Qian J. Transcription factors as readers and effectors of DNA methylation. Nat Rev Genet 2016;17:551-565.

8. Zhong J, Agha G, Baccarelli AA. The Role of DNA Methylation in Cardiovascular Risk and Disease: Methodological Aspects, Study Design, and Data Analysis for Epidemiological Studies. Circ Res $2016 ; 118: 119-131$.

9. Jones PA, Issa JP, Baylin S. Targeting the cancer epigenome for therapy. Nat Rev Genet 2016;17:630-641.

10. Imgenberg-Kreuz J, Carlsson Almlof J, Leonard D, Alexsson A, Nordmark G, Eloranta ML, RantapaaDahlqvist S, Bengtsson AA, Jonsen A, Padyukov L, Gunnarsson I, Svenungsson E, Sjowall C, Ronnblom L, Syvanen AC, Sandling JK. DNA methylation mapping identifies gene regulatory effects in patients with systemic lupus erythematosus. Ann Rheum Dis 2018;77:736-743.

11. Joseph S, George NI, Green-Knox B, Treadwell EL, Word B, Yim S, Lyn-Cook B. Epigenome-wide association study of peripheral blood mononuclear cells in systemic lupus erythematosus: Identifying DNA methylation signatures associated with interferon-related genes based on ethnicity and SLEDAI. J Autoimmun 2019;96:147-157.

12. Webster AP, Plant D, Ecker S, Zufferey F, Bell JT, Feber A, Paul DS, Beck S, Barton A, Williams FMK, Worthington J. Increased DNA methylation variability in rheumatoid arthritis-discordant monozygotic twins. Genome Med 2018;10:64.

13. Zhu H, Wu LF, Mo XB, Lu X, Tang H, Zhu XW, Xia W, Guo YF, Wang MJ, Zeng KQ, Wu J, Qiu YH, Lin X, Zhang YH, Liu YZ, Yi NJ, Deng FY, Lei SF. Rheumatoid arthritis-associated DNA methylation sites in peripheral blood mononuclear cells. Ann Rheum Dis 2019;78:36-42.

14. Hao J, Liu Y, Xu J, Wang W, Wen Y, He A, Fan Q, Guo X, Zhang F. Genome-wide DNA methylation profile analysis identifies differentially methylated loci associated with ankylosis spondylitis. Arthritis Res Ther 2017;19:177.

15. Lai NS, Chou JL, Chen GC, Liu SQ, Lu MC, Chan MW. Association between cytokines and methylation of SOCS-1 in serum of patients with ankylosing spondylitis. Mol Biol Rep 2014;41:3773-3780.

16. Aslani S, Mahmoudi M, Garshasbi M, Jamshidi AR, Karami J, Nicknam MH. Evaluation of DNMT1 gene expression profile and methylation of its promoter region in patients with ankylosing spondylitis. Clin Rheumatol 2016;35:2723-2731.

17. Karami J, Mahmoudi M, Amirzargar A, Gharshasbi M, Jamshidi A, Aslani S, Nicknam MH. Promoter hypermethylation of BCL11B gene correlates with downregulation of gene transcription in ankylosing spondylitis patients. Genes Immun 2017;18:170-175.

18. Saveanu L, Carroll O, Lindo V, Del Val M, Lopez D, Lepelletier Y, Greer F, Schomburg L, Fruci D, Niedermann G, van Endert PM. Concerted peptide trimming by human ERAP1 and ERAP2 aminopeptidase complexes in the endoplasmic reticulum. Nat Immunol 2005;6:689-697.

19. Chang SC, Momburg F, Bhutani N, Goldberg AL. The ER aminopeptidase, ERAP1, trims precursors to lengths of MHC class I peptides by a "molecular ruler" mechanism. Proc Natl Acad Sci U S A 2005;102:1710717112 .

20. van der Linden S, Valkenburg HA, Cats A. Evaluation of diagnostic criteria for ankylosing spondylitis. A proposal for modification of the New York criteria. Arthritis Rheum 1984;27:361-368. 
21. Smith ZD, Chan MM, Humm KC, Karnik R, Mekhoubad S, Regev A, Eggan K, Meissner A. DNA methylation dynamics of the human preimplantation embryo. Nature 2014;511:611-615.

22. Smith ZD, Chan MM, Mikkelsen TS, Gu H, Gnirke A, Regev A, Meissner A. A unique regulatory phase of DNA methylation in the early mammalian embryo. Nature 2012;484:339-344.

23. Ogino S, Nishihara R, Lochhead P, Imamura Y, Kuchiba A, Morikawa T, Yamauchi M, Liao X, Qian ZR, Sun R, Sato K, Kirkner GJ, Wang M, Spiegelman D, Meyerhardt JA, Schernhammer ES, Chan AT, Giovannucci E, Fuchs CS. Prospective study of family history and colorectal cancer risk by tumor LINE-1 methylation level. J Natl Cancer Inst 2013;105:130-140.

24. Kim JJ, Chung SW, Kim JH, Kim JW, Oh JS, Kim S, Song SY, Park J, Kim DH. Promoter methylation of helicase-like transcription factor is associated with the early stages of gastric cancer with family history. Ann Oncol 2006;17:657-662.

25. Pereira MA, Tao L, Wang W, Li Y, Umar A, Steele VE, Lubet RA. Modulation by celecoxib and difluoromethylornithine of the methylation of DNA and the estrogen receptor-alpha gene in rat colon tumors. Carcinogenesis 2004;25:1917-1923.

Figure legend

Figure 1. Results of the association between methylation and mRNA levels of ERAP1 and AS. A) The odds ratio (ORs) and $95 \%$ confidence intervals (CIs) of the methylation 31 CpG loci in ERAP1_1 and ERAP1_2 islands, and six loci in ERAP1_2 island were significantly associated with increased risk of AS. B) The methylation level of ERAP1_1 island was significantly increased in AS patients. C) The methylation level of ERAP1_2 island was significantly increased in AS patients. D) The ROC curves of the methylation levels of ERAP1_1 island, ERAP1_2 island and the combination of all the significantly differential methylated CpG loci. E) The ERAP1 relative expression level was significantly decreased in AS patiens.
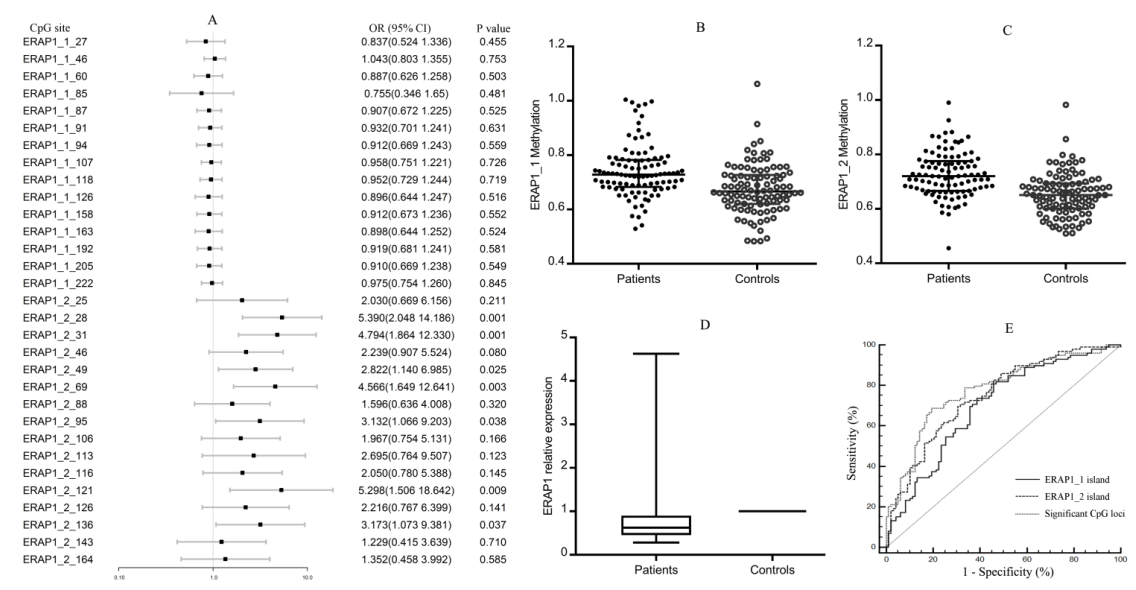

Table 1 Demographic and clinical characteristics of AS patients and HCs

\begin{tabular}{lll}
\hline Variables & Methylation stage & qRT-PCR stage \\
\hline HCs & 99 & 20 \\
Sex ratio $(\mathrm{M} / \mathrm{F})$ & $83 / 16$ & $15 / 5$ \\
Age $($ years) & $31.82 \pm 8.71$ & $31.85 \pm 7.60$ \\
AS patients & 99 & 20 \\
Sex ratio $(\mathrm{M} / \mathrm{F})$ & $77 / 22$ & $14 / 6$ \\
Age (years) & $31.14 \pm 9.93$ & $31.60 \pm 7.86$
\end{tabular}




\begin{tabular}{lll}
\hline Variables & Methylation stage & qRT-PCR stage \\
\hline Body mass index $\left(\mathrm{Kg} / \mathrm{m}^{2}\right)$ & $22.58 \pm 4.36$ & $22.55 \pm 3.03$ \\
Current smoker & $37(37.37)$ & $8(40.00)$ \\
Alcohol use & $25(25.25)$ & $4(20.00)$ \\
Propositus & $63(63.64)$ & $15(80.00)$ \\
Disease duration (years) & $3.00(1.00,8.00)$ & $2.00(1.00,8.50)$ \\
HLA-B27 & $91(91.92)$ & $18(90.00)$ \\
ESR $(\mathrm{mm} / \mathrm{L})$ & $15.50(5.00,32.50)$ & $14.00(7.75,36.75)$ \\
CRP (m/L) & $8.90(2.22,24.62)$ & $6.65(1.15,31.14)$ \\
Ever treatment & $72(75.00)$ & $14(77.78)$ \\
SASP use & $20(20.83)$ & $3(20.00)$ \\
NSAIDs use & $34(35.42)$ & $6(40.00)$ \\
TNFi use & $15(15.63)$ & $9(60.00)$ \\
X-ray classification* & 18 & 14 \\
Level 1 & $2(11.11)$ & $4(28.57)$ \\
Level 2 & $4(22.22)$ & $2(14.29)$ \\
Level 3 & $9(50.00)$ & $7(50.00)$ \\
Level 4 & $3(16.67)$ & $1(7.14)$ \\
Global back pain $(\mathrm{cm})$ & $2.00(1.00,4.00)$ & $0.00(0.00,3.00)$ \\
Finger-floor distance $(\mathrm{cm})$ & $6.00(0.00,19.00)$ & $7.75(0.00,14.75)$ \\
Chest expansion $(\mathrm{cm})$ & $3.00(2.00,5.00)$ & $3.50(2.00,5.50)$ \\
Schober test $(\mathrm{cm})$ & $6.00(4.00,10.00)$ & $7.00(5.00,10.00)$ \\
Occipito wall gap $(\mathrm{cm})$ & $0.00(0.00,2.25)$ & $0.00(0.00,1.50)$ \\
BASFI & $0.90(0.20,2.20)$ & $0.75(0.03,2.48)$ \\
BASDAI & $2.10(1.20,3.50)$ & $2.35(1.88,3.35)$ \\
ASDAS & $3.01(2.10,4.10)$ & $2.74(2.23,4.12)$ \\
\hline
\end{tabular}

* Radiographic record of unilateral; Data were presented as mean \pm standard deviation, median (lower quantile, upper quantile) or number (percent). ASDAS: Ankylosing Spondylitis Disease Activity Score; BASDAI: Bath Ankylosing Spondylitis Disease Activity Index; BASFI: Bath Ankylosing Spondylitis Functional Index; CRP, C-reactive protein; ESR, erythrocyte sedimentation rate; HLA: human leukocyte antigen; M/F: male/female; NSAIDs: non-steroidal anti-inflammatory drugs; SASP: sulfasalazine; TNFi: tumor necrosis factor inhibitor.

Table 2 Detailed methylation status of the 31 CpG sites of AS patients and HCs

\begin{tabular}{llllll}
\hline & Position & AS patients & HCs & $Z$ value & $P$ value \\
\hline ERAP1_1_27 & chr5: 96144043 & $0.7895(0.5712,0.9529)$ & $0.7801(0.5618,0.9698)$ & -0.084 & 0.933 \\
ERAP1_1_46 & chr5: 96144062 & $0.9104(0.6729,1.1268)$ & $0.7168(0.5753,0.8590)$ & -4.25 & $<0.001^{*}$ \\
ERAP1_1_60 & chr5: 96144076 & $0.6360(0.5109,0.8152)$ & $0.5838(0.4167,0.8021)$ & -1.354 & 0.176 \\
ERAP1_1_85 & chr5: 96144101 & $0.5861(0.4308,0.7070)$ & $0.5701(0.4301,0.7692)$ & -0.228 & 0.819 \\
ERAP1_1_87 & chr5: 96144103 & $0.6498(0.4688,0.8114)$ & $0.5820(0.3697,0.7724)$ & -1.218 & 0.223 \\
ERAP1_1_91 & chr5: 96144107 & $0.6407(0.5089,0.8475)$ & $0.6040(0.4004,0.7785)$ & -1.976 & $0.048^{*}$ \\
ERAP1_1_94 & chr5: 96144110 & $0.5208(0.4226,0.7074)$ & $0.4857(0.3252,0.6376)$ & -1.618 & 0.106 \\
ERAP1_1_107 & chr5: 96144123 & $0.7611(0.5875,0.9395)$ & $0.6821(0.4634,0.8755)$ & -2.172 & $0.030^{*}$ \\
ERAP1_1_118 & chr5: 96144134 & $0.9528(0.7246,1.1458)$ & $0.8475(0.6483,1.0695)$ & -2.062 & $0.039^{*}$ \\
ERAP1_1_126 & chr5: 96144142 & $0.7000(0.5629,0.8567)$ & $0.6284(0.4286,0.8375)$ & -1.437 & 0.151 \\
ERAP1_1_158 & chr5: 96144174 & $0.8649(0.6918,1.0172)$ & $0.7955(0.5734,1.0029)$ & -1.383 & 0.167 \\
ERAP1_1_163 & chr5: 96144179 & $0.6275(0.4878,0.7629)$ & $0.5654(0.3956,0.7358)$ & -1.637 & 0.102 \\
ERAP1_1_192 & chr5: 96144208 & $0.6794(0.5039,0.8460)$ & $0.6258(0.3823,0.8313)$ & -1.571 & 0.116
\end{tabular}




\begin{tabular}{llllll}
\hline & Position & AS patients & HCs & $Z$ value & $P$ value \\
\hline ERAP1_1_205 & chr5: 96144221 & $0.9464(0.7389,1.1965)$ & $0.9103(0.6725,1.1808)$ & -0.833 & 0.405 \\
ERAP1_1_222 & chr5: 96144238 & $0.6867(0.5786,0.8371)$ & $0.5356(0.4188,0.7174)$ & -3.75 & $<0.001^{*}$ \\
ERAP1_2_25 & chr5: 96143592 & $0.5988(0.4717,0.8230)$ & $0.6112(0.4119,0.7618)$ & -1.155 & 0.248 \\
ERAP1_2_28 & chr5: 96143595 & $1.0084(0.7770,1.2069)$ & $0.8621(0.6090,1.0695)$ & -3.324 & $0.001^{*}$ \\
ERAP1_2_31 & chr5: 96143598 & $0.9709(0.7394,1.1614)$ & $0.7569(0.6154,1.0467)$ & -3.337 & $0.001^{*}$ \\
ERAP1_2_46 & chr5: 96143613 & $0.8621(0.6250,1.0279)$ & $0.6993(0.5338,0.8897)$ & -3.05 & $0.002^{*}$ \\
ERAP1_2_49 & chr5: 96143616 & $0.7092(0.5754,0.9770)$ & $0.6033(0.4391,0.8621)$ & -3.041 & $0.002^{*}$ \\
ERAP1_2_69 & chr5: 96143636 & $0.8032(0.6220,1.0046)$ & $0.6387(0.4864,0.8137)$ & -3.37 & $0.001^{*}$ \\
ERAP1_2_88 & chr5: 96143655 & $0.6939(0.5000,0.8802)$ & $0.6429(0.4666,0.8850)$ & -0.898 & 0.369 \\
ERAP1_2_95 & chr5: 96143662 & $0.6236(0.4699,0.8486)$ & $0.5302(0.3676,0.7670)$ & -2.379 & $0.017^{*}$ \\
ERAP1_2_106 & chr5: 96143673 & $0.7472(0.4994,0.9379)$ & $0.7164(0.4593,0.9050)$ & -0.987 & 0.324 \\
ERAP1_2_113 & chr5: 96143680 & $0.5856(0.4579,0.7128)$ & $0.4888(0.3643,0.7319)$ & -1.903 & 0.057 \\
ERAP1_2_116 & chr5: 96143683 & $0.6780(0.5025,0.8641)$ & $0.6047(0.4269,0.8403)$ & -1.656 & 0.098 \\
ERAP1_2_121 & chr5: 96143688 & $0.5879(0.4414,0.7075)$ & $0.4707(0.3286,0.6745)$ & -2.937 & $0.003^{*}$ \\
ERAP1_2_126 & chr5: 96143693 & $0.6873(0.4878,0.8488)$ & $0.6545(0.4255,0.7850)$ & -1.284 & 0.199 \\
ERAP1_2_136 & chr5: 96143703 & $0.6790(0.5093,0.8418)$ & $0.5970(0.3918,0.7364)$ & -2.536 & $0.011^{*}$ \\
ERAP1_2_143 & chr5: 96143710 & $0.6154(0.4202,0.7618)$ & $0.5396(0.4008,0.7353)$ & -0.944 & 0.345 \\
ERAP1_2_164 & chr5: 96143731 & $0.5904(0.4409,0.7687)$ & $0.5464(0.3774,0.7856)$ & -0.764 & 0.445 \\
\hline
\end{tabular}

${ }^{*} P<0.05 ;$ chr: chromosome.

Table 3 The diagnosis value of methylation of ERAP1 gene

\begin{tabular}{llllll}
\hline & AUC $(95 \% \mathrm{CI})$ & Sensitivity $(\%)$ & Specificity $(\%)$ & Cutoff point & $P$ value \\
\hline ERAP1_1 & $0.669(0.626,0.707)$ & 0.818 & 0.531 & 0.671 & $P<0.001^{*}$ \\
ERAP1_2 & $0.750(0.683,0.818)$ & 0.707 & 0.684 & 0.681 & $P<0.001^{*}$ \\
CpG loci $\#$ & $0.779(0.714,0.844)$ & 0.717 & 0.737 & 0.500 & $P<0.001^{*}$ \\
\hline
\end{tabular}

${ }^{*} P<0.05$; $\#$ the combined diagnosis of 15 differential methylated CpG loci.

Table 4 Subgroup analysis of methylation levels of CpG islands of ERAP1

\begin{tabular}{|c|c|c|c|c|c|c|}
\hline Group & ERAP1_1 & ERAP1_1 & ERAP1_1 & ERAP1_2 & ERAP1_2 & ERAP1_2 \\
\hline & Median (Quartiles) & $\mathrm{Z}$ value & $P$ value & Median (Quartiles) & $Z$ value & $P$ value \\
\hline Male & $0.7228(0.6768,0.7675)$ & -1.831 & 0.067 & $0.7204(0.6724,0.7689)$ & -0.412 & 0.680 \\
\hline Female & $0.7463(0.7154,0.7985)$ & & & $0.7153(0.6639,0.8289)$ & & \\
\hline HLA-B27 positive & $0.7285(0.6835,0.7807)$ & -0.180 & 0.857 & $0.7204(0.6661,0.7840)$ & -1.040 & 0.298 \\
\hline HLA-B27 negative & $0.7433(0.6749,0.7898)$ & & & $0.7045(0.6410,0.7475)$ & & \\
\hline Smoker & $0.7299(0.6732,0.7923)$ & -0.268 & 0.789 & $0.7418(0.6633,0.7911)$ & -0.839 & 0.401 \\
\hline Non-smoker & $0.7266(0.6877,0.7812)$ & & & $0.7089(0.6705,0.7748)$ & & \\
\hline Alcohol use & $0.7085(0.6732,0.7768)$ & -0.969 & 0.332 & $0.6867(0.6593,0.7524)$ & -1.608 & 0.108 \\
\hline Without alcohol use & $0.7299(0.6893,0.7821)$ & & & $0.7245(0.6729,0.7833)$ & & \\
\hline Propositus & $0.7322(0.6914,0.7913)$ & -2.258 & $0.024^{*}$ & $0.7241(0.6738,0.7923)$ & -1.336 & 0.182 \\
\hline Non-proprsitus & $0.7014(0.6671,0.7428)$ & & & $0.7140(0.6609,0.7643)$ & & \\
\hline Ever treatment & $0.7322(0.6790,0.7873)$ & -0.605 & 0.545 & $0.7265(0.6770,0.7797)$ & -0.753 & 0.451 \\
\hline Without treatment & $0.7246(0.6956,0.7354)$ & & & $0.6970(0.6624,0.7850)$ & & \\
\hline SASP & $0.7768(0.6909,0.8041)$ & -1.331 & 0.183 & $0.7498(0.7007,0.7923)$ & -1.361 & 0.173 \\
\hline Without SASP & $0.7252(0.6790,0.7651)$ & & & $0.7177(0.6631,0.7688)$ & & \\
\hline
\end{tabular}




\begin{tabular}{lllllll}
\hline Group & ERAP1_1 & ERAP1_1 & ERAP1_1 & ERAP1_2 & ERAP1_2 & ERAP1_2 \\
\hline NSAIDs & $0.7548(0.7007,0.7889)$ & -2.113 & $0.035^{*}$ & $0.7252(0.6821,0.7859)$ & -0.537 & 0.591 \\
Without NSAIDs & $0.7195(0.6747,0.7645)$ & & & $0.7201(0.6638,0.7713)$ & & \\
TNFi & $0.7299(0.6934,0.7687)$ & -0.095 & 0.924 & $0.7092(0.6661,0.7555)$ & -0.838 & 0.402 \\
Without TNFi & $0.7261(0.6753,0.7859)$ & & & $0.7222(0.6681,0.7909)$ & & \\
\hline
\end{tabular}

* $P<0.05$; HLA: human leukocyte antigen; NSAIDs: non-steroidal anti-inflammatory drugs; SASP: sulfasalazine; TNFi: tumor necrosis factor inhibitor.

Table 5 Association analysis of methylation levels of $\mathrm{CpG}$ islands and mRNA level of ERAP1

\begin{tabular}{|c|c|c|c|c|c|c|}
\hline & ERAP1_1 & ERAP1_1 & ERAP1_2 & ERAP1_2 & $\begin{array}{l}\text { ERAP1 } \\
\text { mRNA }\end{array}$ & $\begin{array}{l}\text { ERAP1 } \\
\text { mRNA }\end{array}$ \\
\hline & $\mathrm{r}_{\mathrm{s}}$ & $P$ value & $\mathrm{r}_{\mathrm{s}}$ & $P$ value & $\mathrm{r}_{\mathrm{s}}$ & $P$ value \\
\hline Age & -0.085 & 0.400 & -0.018 & 0.856 & -0.271 & 0.247 \\
\hline $\begin{array}{l}\text { Body mass } \\
\text { index }\end{array}$ & -0.156 & 0.127 & -0.232 & 0.022 & -0.659 & 0.002 \\
\hline $\begin{array}{l}\text { Disease } \\
\text { duration }\end{array}$ & -0.020 & 0.851 & 0.186 & 0.071 & 0.068 & 0.774 \\
\hline ESR & 0.016 & 0.878 & -0.047 & 0.647 & -1.126 & 0.620 \\
\hline $\mathrm{CRP}$ & -0.010 & 0.923 & 0.001 & 0.995 & -0.096 & 0.724 \\
\hline $\begin{array}{l}\text { X-ray } \\
\text { classification* }\end{array}$ & 0.007 & 0.979 & 0.548 & 0.018 & 0.179 & 0.541 \\
\hline $\begin{array}{l}\text { Global back } \\
\text { pain }\end{array}$ & 0.502 & 0.002 & -0.222 & 0.194 & 0.137 & 0.576 \\
\hline $\begin{array}{l}\text { Finger-floor } \\
\text { distance }\end{array}$ & -0.054 & 0.608 & -0.107 & 0.320 & -0.075 & 0.752 \\
\hline $\begin{array}{l}\text { Chest } \\
\text { expansion }\end{array}$ & 0.045 & 0.679 & -0.107 & 0.320 & 0.697 & 0.001 \\
\hline Schober test & 0.127 & 0.229 & -0.009 & 0.935 & 0.537 & 0.018 \\
\hline $\begin{array}{l}\text { Occipito wall } \\
\text { gap }\end{array}$ & -0.020 & 0.847 & -0.054 & 0.608 & -0.010 & 0.967 \\
\hline BASFI & -0.124 & 0.223 & -0.095 & 0.349 & 0.001 & 0.997 \\
\hline BASDAI & -0.084 & 0.411 & -0.210 & 0.073 & -0.092 & 0.700 \\
\hline ASDAS & -0.039 & 0.703 & -0.150 & 0.142 & -0.221 & 0.428 \\
\hline
\end{tabular}

* Radiographic record of unilateral; ASDAS: Ankylosing Spondylitis Disease Activity Score; BASDAI: Bath Ankylosing Spondylitis Disease Activity Index; BASFI: Bath Ankylosing Spondylitis Functional Index; CRP, C-reactive protein; ESR, erythrocyte sedimentation rate. $r_{s}$ : Spearman's rank correlation coefficient 


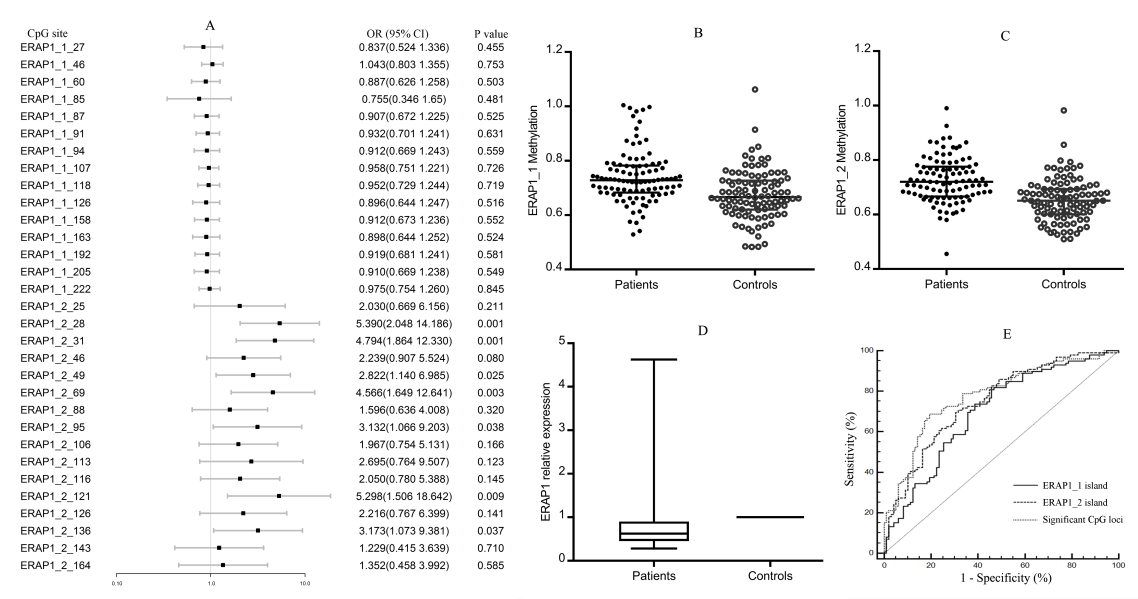

